\title{
COMPUTING WITH WORDS WITH THE USE OF INVERSE RDM MODELS OF MEMBERSHIP FUNCTIONS
}

\author{
ANDRZEJ PIEGAT ${ }^{a}$, MARCIN PLUCIŃSKI $^{a, *}$ \\ ${ }^{a}$ Faculty of Computer Science and Information Technology \\ West Pomeranian University of Technology, Żołnierska 49, 71-210 Szczecin, Poland \\ e-mail: \{apiegat, mplucinski\}@wi.zut.edu.pl
}

\begin{abstract}
Computing with words is a way to artificial, human-like thinking. The paper shows some new possibilities of solving difficult problems of computing with words which are offered by relative-distance-measure RDM models of fuzzy membership functions. Such models are based on RDM interval arithmetic. The way of calculation with words was shown using a specific problem of flight delay formulated by Lotfi Zadeh. The problem seems easy at first sight, but according to the authors' knowledge it has not been solved yet. Results produced with the achieved solution were tested. The investigations also showed that computing with words sometimes offers possibilities of achieving better problem solutions than with the human mind.
\end{abstract}

Keywords: computing with words, fuzzy arithmetic, RDM fuzzy arithmetic, granular computing.

\section{Introduction}

Computing with words $(\mathrm{CwW})$ is an interesting way to creating an artificial thinking of an artificial computer brain that would be able to solve problems on the basis of linguistic information provided. The idea of $\mathrm{CwW}$ created by Lotfi Zadeh has been presented in scientific journals after 1990 (Zadeh, 1996a; 1996b; 2001; 2002; 2004; 2005; 2006a; 2006b; 2009, Zadeh and Kacprzyk, 1999). From the very beginning, CwW has been connected with fuzzy and interval arithmetic (Hansen, 1975; Kaufmann and Gupta, 1991; Piegat, 2001; Hanss, 2005; Tomaszewska, 2014), with granular computing (Batyrshin, 2002; Pedrycz and Gomide, 2007; Aliev et al., 2012; Piegat and Landowski, 2013a), with human-centric computing (Pedrycz and Gomide, 2007), with data mining, database querying and data analysis (Kacprzyk and Zadrożny, 1999; 2002; 2010; Zadeh and Kacprzyk, 1999; Grzegorzewski and Hryniewicz, 2002; Batyrshin and Wageknecht, 2002), and with plant control (Zhou,

\footnotetext{
* Corresponding author

In 2014, Professor Lotfi Zadeh, the inventor of fuzzy sets and the idea of computing with words, celebrated his 93rd birthday. On this occasion, the 4th World Conference on Soft Computing, dedicated to his research heritage, was organized by UC Berkeley. The authors of this paper dedicate it to Professor Zadeh, also with reference to a special issue of the International Journal of Applied Mathematics and Computer Science (Zadeh, 2002).
}

2002). CwW is very important for decision-making, where very often, apart from numerical data, linguistic information provided by a problem expert is at our disposal (Herrera et al., 1999; Mendel, 2002; Aliev et al., 2012). In the frame of $\mathrm{CwW}$ there are also made various basic investigations concerning scientific tools facilitating its application (Gemeinder, 2002; De Cock and Kerre, 2002; Cao, 2003; Lawry, 2006; Türkşen, 2007).

Using linguistic and numerical information together enables more effective decision-making than using only numerical one. Additionally, the linguistic information provided by the problem expert can be much more important and informative for problem solving than numerical data, because the accuracy of numerical data can be sometimes very low, although the data may appear highly precise. For example, the statement published by a statistical office that the official import to a certain country equals \$ 9928327335 may seem a very precise information piece. However, according to experts, the real import to the country is about $20 \%$ greater because of its illegal component.

$\mathrm{CwW}$ is a rather difficult mathematical area and at present its development phase can be evaluated as an initial state. This opinion is supported by the fact that practical possibilities of the present $\mathrm{CwW}$ are rather limited. Professor Lotfi Zadeh, 
the creator of the $\mathrm{CwW}$ idea, formulated many challenge problems. These problems can be found in his publications and on his website at http: / / www.cs.berkeley.edu/ zadeh/. They represent one of the many areas of $\mathrm{CwW}$ that can be called 'autonomous thinking'. It greatly differs from, e.g., fuzzy control. Further on, several examples are presented (Zadeh, 1996b):

- Tall Swedes

Most Swedes are tall. How many are short? What is the average height of Swedes?

\section{- Temperature}

Usually the temperature in my city is not very low and not very high. What is the average temperature?

\section{- Flight delay}

Usually most United Airlines flights from San Francisco leave on time. What is the probability that my flight will be delayed?

\section{- Balls in a box}

A box contains about 20 balls of various sizes. Most are large. What is the number of small balls? What is the probability that a ball drawn at random is neither small nor large?

The above problems may seem very easy for non-specialists. However, it is not true. Many persons cannot solve them and such an ability may be very important for autonomous thinking. If simple $\mathrm{CwW}$ examples cannot be solved, then how could more complex tasks be? Such problems have been frequently repeated and discussed on the BISC forum (a discussion forum at UC Berkeley conducted by Professor Lotfi Zadeh). They are examples of autonomous thinking or Computing with Words in Zadeh's sense. Solving such 'trivial' problems paves a way to more complicated $\mathrm{CwW}$ problems, to realisation of dreams and plans of Professor Zadeh and thousands of other scientists.

Specific solutions of $\mathrm{CwW}$ problems are in the scientific literature rather rare. One of the reasons can be limited possibilities of the present fuzzy arithmetic and mathematics that were carefully commented, e.g., by Dymova (2011). Difficulties in application of this arithmetic are caused by the impossibility of taking into account couplings (full or partial) and dependences existing between variables or also between linguistic values of a single variable, by the impossibility of solving even simple equations, or by the phenomenon of entropy increasing of a solution. Therefore, the paper of Aliev et al. (2012) has generated our deep interest because it presents specific solutions of Zadeh's two $\mathrm{CwW}$ problems known as the two-boxes problem and the investment problem. Both were solved with the application of fuzzy arithmetic and mathematics. In the works of Rajati et al. (2011) as well as Rajati and Mendel (2012), Zadeh's challenge problem of tall Swedes has been solved with two methods. These papers show how complicated the problems of autonomous thinking are. They require considerable theoretical knowledge. Therefore, industrial engineers, common economists or physicians may have difficulties with its application. We present a less complicated method of $\mathrm{CwW}$ that is based on RDM models of membership functions and on multidimensional RDM interval arithmetic (RDM-IA) (Piegat and Landowski, 2012; 2013a; 2013b; Piegat and Tomaszewska, 2013; Tomaszewska and Piegat, 2014). This arithmetic differs considerably from Moore's interval arithmetic (Moore, 1966; Moore et al., 2009). Further on, the idea of the multidimensional RDM-IA will be shortly presented.

\section{Idea of multidimensional RDM interval arithmetic}

We do not claim that Moore's interval arithmetic is generally incorrect, because it properly realises basic arithmetic operations such as addition, subtraction, division and multiplication, although in a simplified one-dimensional way and without taking into account dependences between variables and between particular intervals that can exist in real problems. However, this arithmetic causes many paradoxes that are described in the literature, e.g., by Sevastjanov and Dymova (2009) or Dymova (2011). Some faults of Moore's interval arithmetic are

(a) the excess width effect,

(b) the dependency problem,

(c) difficulties with solving even simplest interval equations,

(d) problems with the of right-hand sides of the interval equations,

(e) absurd solutions and requests to introduce negative entropy into the system.

Problems (c) and (e) will be illustrated with an example of an equation with one unknown quantity. Consider the interval equation

$$
\begin{aligned}
& {[\underline{a}, \bar{a}]+[\underline{x}, \bar{x}]=[\underline{c}, \bar{c}],} \\
& {[1,3]+[\underline{x}, \bar{x}]=[3,4] .}
\end{aligned}
$$

It can be solved directly in the following way:

$$
\begin{array}{ll}
1+\underline{x}=3, & \underline{x}=2, \\
3+\bar{x}=4, & \bar{x}=1 .
\end{array}
$$



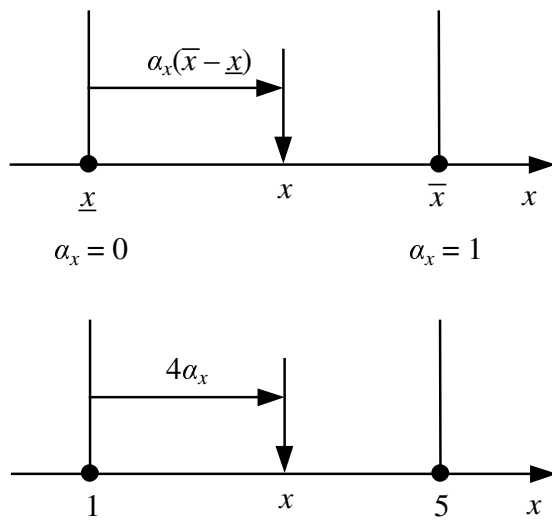

$\alpha_{x}=0$

$\alpha_{x}=1$

Fig. 1. Example of the internal variable $\alpha$ of the interval, which has the meaning of a relative-distance-measure.

Finally, we obtain a solution which is absurd because $\underline{x}>$ $\bar{x}$.

Equation (1) can also be solved in another way:

$$
[\underline{x}, \bar{x}]=[3,4]-[1,3]=[0,3] .
$$

However, such a solution does not satisfy Eqn. (1) since, after inserting it into the initial formula, we get

$$
[1,3]+[0,3] \neq[3,4] .
$$

Multidimensional RDM arithmetic introduces an internal variable $\alpha \in[0,1]$, which has the meaning of a relative-distance-measure (Fig. 1).

The aim of introducing RDM variables is not to make unnecessary parameterization of intervals, but to introduce the Cartesian coordinate system in interval arithmetic, similarly as in the conventional crisp arithmetic, where it has been used for centuries. Observe that Moore's arithmetic uses in calculations only extremities of intervals. Their interiors do not take part in calculations. This means a kind of arithmetic 'vacuum'. Let us consider the subtraction $A-C=X=[3,4]-$ $[1,3]=[0,3]$, realised according to Moore's arithmetic. It is visualised in Fig. 2. If we make addition $X+C=$ $[0,3]+[1,3]$, then we will get the interval $[1,6]$, not $A=$ $[3,4]$. Why? Because the 'result' $[0,3]$ is not a complete result but only its span, which is shown in Fig. 2. Minima and maxima of mathematical functions not always lie on borders of function domains. They frequently lie inside these domains. In such a case, extrema cannot be detected by Moore's arithmetic.

Thanks to $\alpha$ 's RDM variables, RDM arithmetic introduces a local Cartesian coordinate system in the problem domain, which makes the interiors of interval no more arithmetic vacuums and they can now take part in calculations. Thanks to it, in more complicated problems, if extrema lie inside their domains, they can

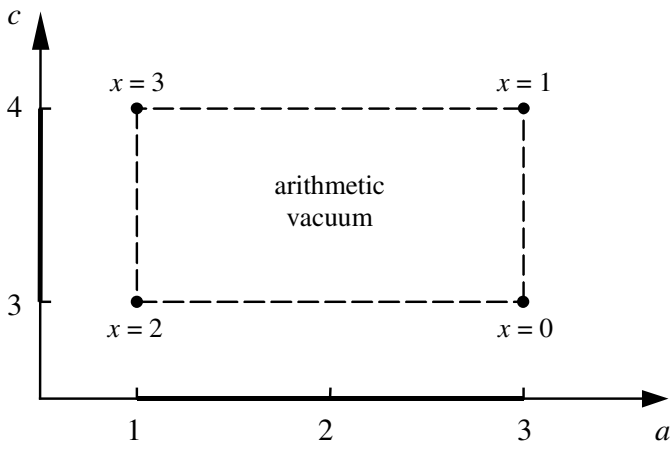

(a)

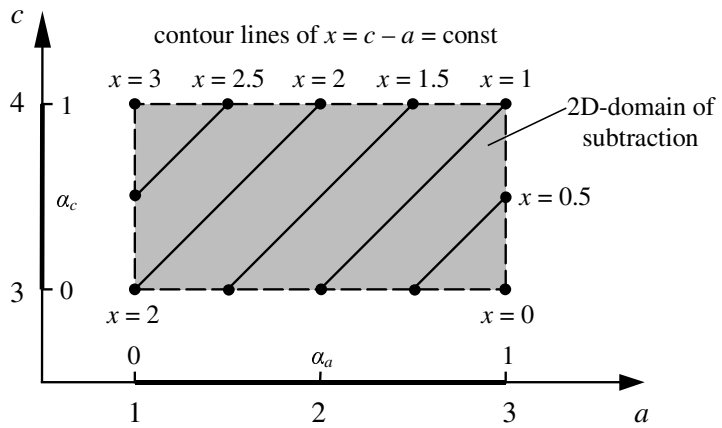

(b)

Fig. 2. Visualization of the interval subtraction $C-A=$ $X=[3,4]-[1,3]$ : Moore's arithmetic (a), RDM arithmetic (b).

be detected by usual function examination, similarly as in the conventional crisp mathematics. Moore's arithmetic detects extrema only if they lie at the boundary of the solution space as in the case of all monotonic operations such as addition, subtraction, multiplication and division.

RDM arithmetic has almost the same mathematical properties as the conventional one (Landowski, 2014). Let $A, B, C$ be intervals. Points $1-7$ below present the most important properties of RDM arithmetic:

1. $A+B=B+A, A B=B A$ : commutativity laws of addition and multiplication.

2. $A+(B+C)=(A+B)+C, A(B C)=(A B) C$ : associativity laws of addition and multiplication.

3. For each $A$ in $R$ there exists $-A$ in $R$ such that $A+$ $(-A)=(-A)+A=0 .-A$ is the additive inverse of $A$.

4. $A(B+C)=(A B)+(A C)$ : left distributivity law, $(B+C) A=(B A)+(C A)$ : right distributivity law.

5. For each $A$ in $R, 0 \notin A$, there exists $A^{-1}=1 / A$ in $R$ such that $A A^{-1}=A(1 / A)=1 . A^{-1}$ is the multiplicative inverse of $A$. 
6. $A+C=B+C \Rightarrow A=B$ : cancellation law of addition.

7. $C A=C B \Rightarrow A=B$ : cancellation law of multiplication.

In the case of Moore's arithmetic, Laws 3, 4, 5, 7 do not hold (Moore et al., 2009)! Consequently, transformations of formulas cannot be made. For example, in the equation $A+X=C$, shifting $A$ to the right-hand side to get $X=C-A$ is not allowed because Law 3 does not hold. Because some transformations are not allowed, more complicated algebraic and mathematical problems cannot be solved. Checking whether particular laws 1-7 hold for RDM arithmetic is easy. In the formulas for the laws, RDM models of intervals $A, B, C$ should be inserted and the law equations should be examined (Landowski, 2014). Frequently, the question is asked: "What are practical benefits of RDM arithmetic?" These are mentioned below:

(a) Complicated problems can be solved, thanks to the possibility of transforming equations.

(b) Almost all laws of the arithmetic of crisp numbers hold for RDM arithmetic.

(c) RDM arithmetic provides complete, multidimensional problem solutions from which various simplified representations such as cardinality distribution, a span of a solution (Moore's solution) or a center of gravity can be derived.

The third benefit will be explained using the example of an interval addition $A+B=C$, where $A=[1,2]$, $B=[2,4]$. In terms of RDM arithmetic, intervals are modeled with the use of RDM variables:

$$
\begin{aligned}
& A: a=1+\alpha_{a}, \quad B: b=2+2 \alpha_{b}, \\
& c=a+b=3+\alpha_{a}+2 \alpha_{b}, \quad \alpha_{a}, \alpha_{b} \in[0,1] .
\end{aligned}
$$

The complete solution set $C$ is not a $1 \mathrm{D}$ interval but a $3 \mathrm{D}$ information granule determined by (2) and shown in Fig. 3.

In Fig. 3, contour lines of constant addition results ( $c=a+b=$ const) can be seen. The lengths of these lines (segments) are measures of the cardinalities of particular solution sets (e.g., of $c=5=$ const). The distribution of the cardinality of particular solution sets $c=$ const, shown in Fig. 4 is a valuable two-dimensional representation of the complete solution $c=a+b=3+\alpha_{a}+2 \alpha_{b}, \alpha_{a}, \alpha_{b} \in$ $[0,1]$.

The next representation of the complete solution set from Fig. 3 can be the $\operatorname{span}[\min (c), \max (c)]=[\underline{c}, \bar{c}]=$ $[3,6]$ shown in Fig. 5. The span $[\underline{c}, \bar{c}]=[3,6]$ can be achieved by usual function examination of the complete

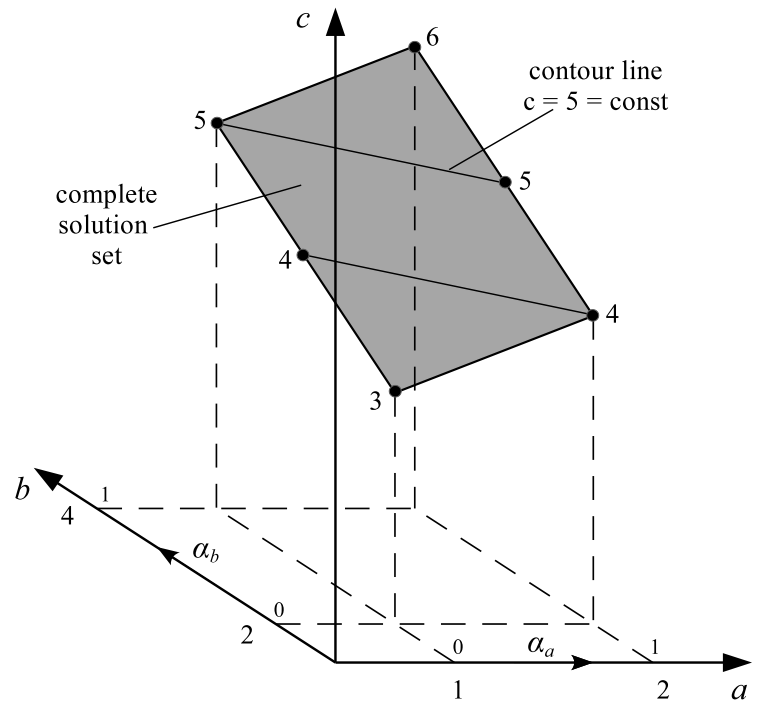

Fig. 3. Complete 3-dimensional result (solution set) $C: c=$ $a+b=3+\alpha_{a}+2 \alpha_{b}, \alpha_{a}, \alpha_{b} \in[0,1]$ of the interval addition $A+B=[1,2]+[2,4]$, achieved with the use of RDM arithmetic.

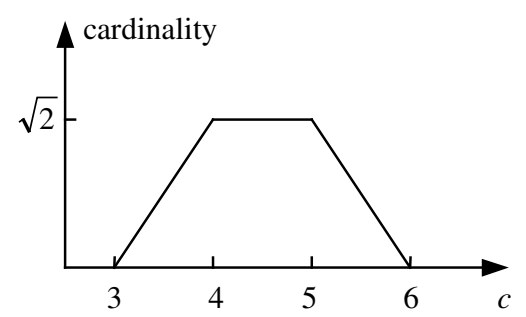

Fig. 4. Cardinality distribution as a representation of the complete, 3D solution set $C: c=a+b=3+\alpha_{a}+$ $2 \alpha_{b}, \alpha_{a}, \alpha_{b} \in[0,1]$.

solution set $C: c=a+b=3+\alpha_{a}+2 \alpha_{b}, \alpha_{a}, \alpha_{b} \in[0,1]$. It is easy to determine that $\min (c)=\underline{c}=3$ is achieved for $\alpha_{a}=\alpha_{b}=0$ and $\max (c)=\bar{c}=6$ for $\alpha_{a}=\alpha_{b}=1$. The last and simplest representation of the complete solution set can be its center of gravity (CofG) shown in Fig.6

To solve Eqn. (11), we can use two RDM variables: $\alpha_{a}$ and $\alpha_{c}$. Then the interval $[\underline{a}, \bar{a}]=[1,3]$ takes the form $a=1+2 \alpha_{a}, \alpha_{a} \in[0,1]$, and the interval $[\underline{c}, \bar{c}]=[3,4]$ takes the form $c=3+\alpha_{c}, \alpha_{c} \in[0,1]$. Now, Eqn. (1) can be rewritten according to the rules of RDM arithmetic as

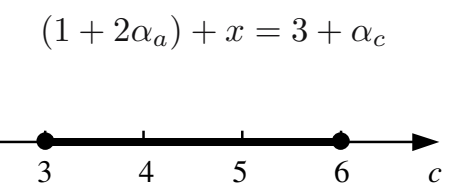

Fig. 5. Span $[3,6]$ of the complete RDM solution set $C: c=$ $a+b=3+\alpha_{a}+2 \alpha_{b}, \alpha_{a}, \alpha_{b} \in[0,1]$ being Moore's solution and the $1 \mathrm{D}$ representation of the interval addition $A+B=C$. 


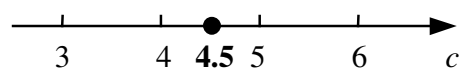

Fig. 6. Simplest representation of the complete solution set $C$ : $c=a+b=3+\alpha_{a}+2 \alpha_{b}, \alpha_{a}, \alpha_{b} \in[0,1]$ of the interval addition $A+B$ in the form of its center of gravity.

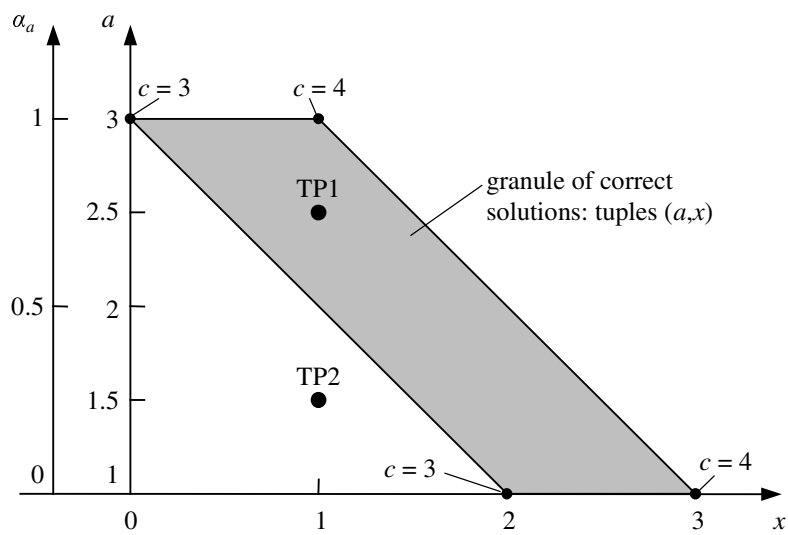

Fig. 7. Solution granule of the equation $[1,3]+[\underline{x}, \bar{x}]=[3,4]$ consisting of pairs $(a, x)$ satisfying the condition (3). TP1 and TP2 are test points.

and

$$
x=2-2 \alpha_{a}+\alpha_{c}, \quad \alpha_{a} \in[0,1], \alpha_{c} \in[0,1] .
$$

It should be noted that the solution $x$ depends on two variables: $\alpha_{a}$ and $\alpha_{c}$. Thus, this solution is not one-dimensional as Moore's arithmetic suggests, but two-dimensional one. The solution is presented in Fig. 7 , and values from the figure are additionally explained in Table 1

The presented example shows that, in a general case, it is impossible to form a one-dimensional solution $[\underline{x}, \bar{x}]$ of interval equations. This is caused by the fact that solutions to problems with data uncertainty are multidimensional.

The correctness of each interval arithmetic can be checked with test points. There are two test points in Fig. 77. TP1 $(a=2.5, x=1)$ and TP2 $(a=1.5, x=1)$. The reader may also use other, individually chosen test points. Substituting the point TP2 $(1.5,1)$ into the equation $a+x=c$, we obtain

$$
1.5+1=2.5 \notin[3,4],
$$

Table 1. Values of variables $a$ and $c$ for boundary values of RDM variables $\alpha_{a}$ and $\alpha_{c}$.

\begin{tabular}{|c||cccc|}
\hline$\alpha_{a}$ & 0 & 0 & 1 & 1 \\
$a$ & 1 & 1 & 3 & 3 \\
\hline$\alpha_{c}$ & 0 & 1 & 0 & 1 \\
$c$ & 3 & 4 & 3 & 4 \\
\hline$x$ & 2 & 3 & 0 & 1 \\
\hline
\end{tabular}

so the solution does not satisfy the interval equation $[1,3]+[\underline{x}, \bar{x}]=[3,4]$. Substituting the coordinates of the point TP1 $(2.5,1)$, we obtain the correct solution of the interval equation:

$$
2.5+1=3.5 \in[3,4]
$$

The above example shows that the notation of the interval equation

$$
[a]+[x]=[c],
$$

which is suggested by Moore's arithmetic and which is commonly used, is incorrect and leads to incorrect inference. The correct notation should have the form

$$
\begin{aligned}
& {[\underline{a}, \bar{a}]+[\underline{x}(a), \bar{x}(a)]=[\underline{c}, \bar{c}],} \\
& {[1,3]+[\underline{x}=3-a, \bar{x}=4-a]=[3,4],}
\end{aligned}
$$

because the values of $x$ and $a$ are usually partly dependent (see Fig.7).

In interval calculations, all known intervals form a hyper-rectangular knowledge granule. In the case of Eqn. (1) it is a $2 \mathrm{D}$ granule $[1,3] \times[3,4]$. The solution of the discussed interval equation is also the 2D granule $[\underline{x}=3-a, \bar{x}=4-a]$ shown in Fig. 7 The multidimensional, non-regular, not hyper-rectangular solution granule cannot be simplified to a one-dimensional interval $[\underline{x}, \bar{x}]$. A $1 \mathrm{D}$ interval can only inform about a spread of the multidimensional solution, which is simplified information about the full solution. In the case of Eqn. (1) such a simplified representation of the full solution is the interval $[\underline{x}, \bar{x}]=[0,3]$.

Further capabilities that are offered by multidimensional RDM interval arithmetic are described by Piegat and Landowski (2012; 2013a). Forthoming publications are under review in several international journals.

\section{Solution of Zadeh's flight delay problem using RDM models of inverse membership functions}

First, let us recall the problem. We have obtained the information "Usually most United Airlines flights from San Francisco leave on time" and we should evaluate "What is the probability that my flight (today) will be delayed?". Knowledge about the problem has been provided by a perceptional information source (PIS) on the basis of long observations of the airport (Zadeh, 1996b). Assume that the PIS understands the linguistic quantifier most (Zadeh, 2001) in the way expressed by a membership function shown in Fig. 8. The quantifier most concerns the ratio of airplanes leaving the airport punctually on a single day. Therefore, the variable $m$ has 


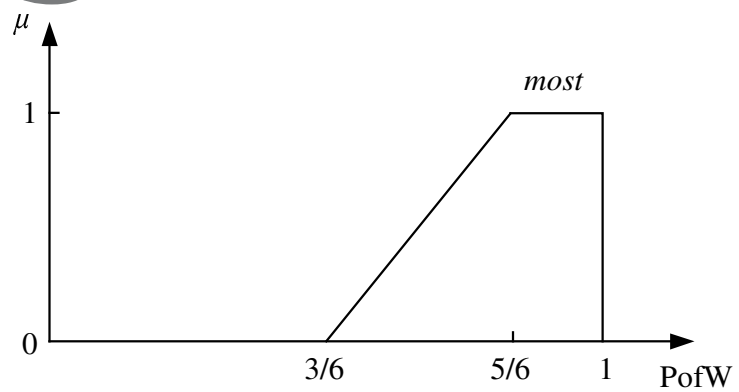

a)

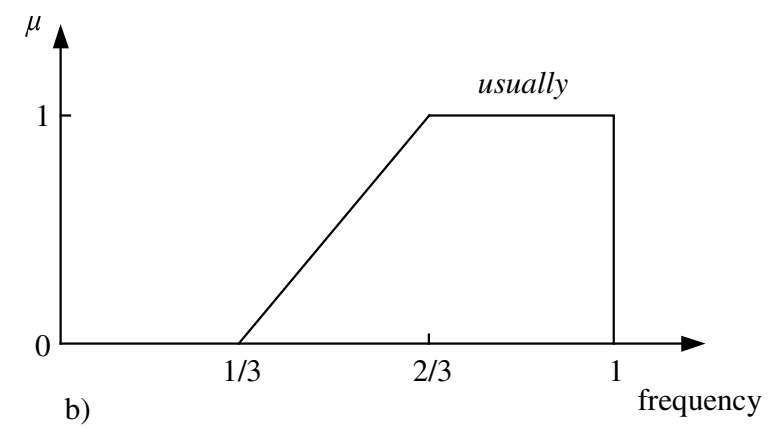

Fig. 8. Membership function of the linguistic quantifiers most (a) and usually (b).

the sense of part of the whole (PofW). The quantifier $u s u$ ally concerns the ratio of these days in the whole number of observed days when most airplanes of United Airlines departed punctually. Thus, the variable $u$ has a sense of relative frequency.

Further on, a formula will be derived that defines an inverse membership function $x=f\left(\mu, \alpha_{x}\right), \alpha_{x} \in$ $[0,1]$, for the trapezium function, Fig. 9, which is a generalization of other function types, e.g., of the triangle function.

The left-hand side of the trapezium function is determined by

$$
x_{L}=a+(b-a) \mu,
$$

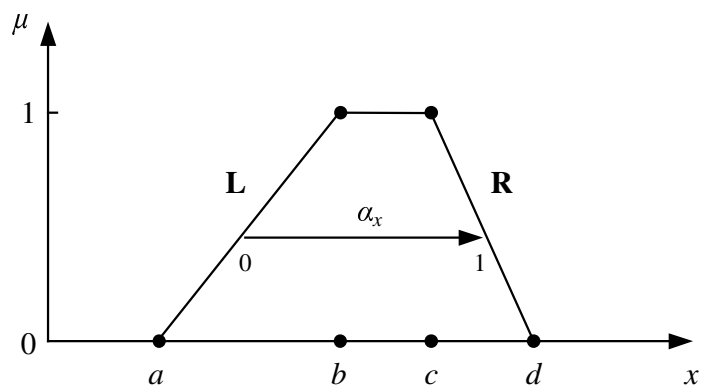

Fig. 9. Trapezium membership function: L-left-hand side, $\mathrm{R}$-right-hand side, $\alpha_{x} \mathrm{RDM}$ variable equal to zero on the left-hand side and to one on the right-hand side of the function, $\alpha_{x} \in[0,1]$.

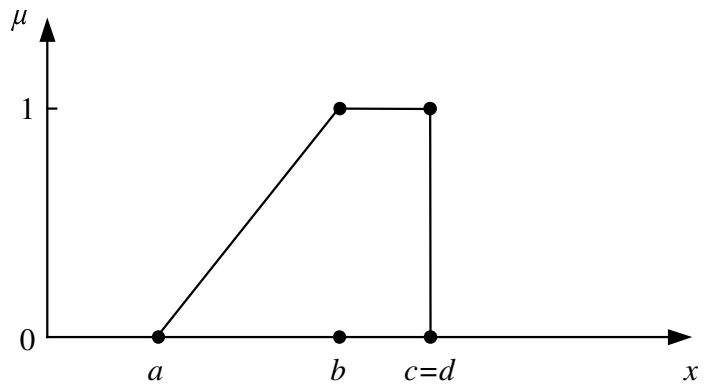

Fig. 10. Right-border trapezium membership function.

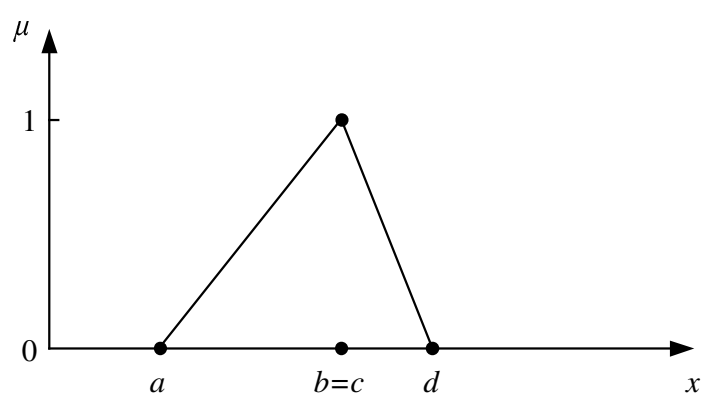

Fig. 11. Triangle membership function.

and the right-hand side by

$$
x_{R}=d-(d-c) \mu .
$$

Thus the full, inverse trapezium function with its interior is determined by the formulas

$$
\begin{aligned}
& x=x_{L}+\left(x_{R}-x_{L}\right) \alpha_{x}, \quad \alpha_{x} \in[0,1], \\
& x=[a+(b-a) \mu]+[(d-a)-\mu(b+d-a-c)] \alpha_{x} .
\end{aligned}
$$

For the case of border trapezium functions as most and usually, Fig. 10, the formula (8) takes the form

$$
\begin{array}{r}
x=[a+(b-a) \mu]+[(c-a)-\mu(b-a)] \alpha_{x}, \\
\alpha_{x} \in[0,1] .
\end{array}
$$

In the case of the inverse triangle membership function, Fig. 11, the RDM model (8) takes the form

$$
\begin{array}{r}
x=[a+(b-a) \mu]+(d-a)(1-\mu) \alpha_{x}, \\
\alpha_{x} \in[0,1] .
\end{array}
$$

In the flight delay problem, the linguistic quantifier most (Zadeh, 2004) occurs (Fig. 12). Its function can be defined by the inverse RDM model (11) containing a variable $m$. This variable can be interpreted as an indication of 'part of the whole'. Its value, e.g., $m=$ 0.70 , means $70 \%$ of United Airlines aeroplanes that depart punctually on a single day. The linguistic quantifier 


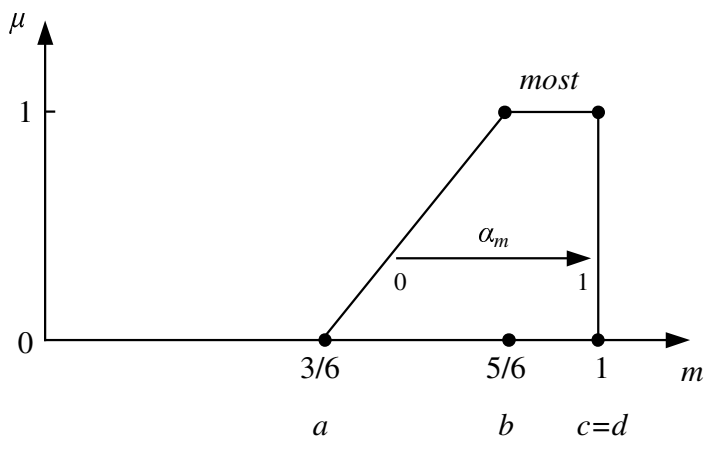

Fig. 12. Trapezium right-border membership function most occurring in the flight delay problem.

most contains in its support all numerical values of $m \in$ $[0.5,1]$

most : $m=\frac{1}{6}\left[(3+2 \mu)+(3-2 \mu) \alpha_{m}\right], \quad \alpha_{m} \in[0,1]$.

Formulas for various $\mu$-cuts of this three-dimensional function can be achieved after inserting into the formula (11) specific values of membership $\mu$ :

$$
\begin{aligned}
\mu=1: & m(\mu=1)=\frac{1}{6}\left(5+\alpha_{m}\right), \quad \alpha_{m} \in[0,1], \\
\mu=0.5: & m(\mu=0.5)=\frac{1}{6}\left(4+2 \alpha_{m}\right), \\
\mu=0: & m(\mu=0)=\frac{1}{6}\left(3+3 \alpha_{m}\right) .
\end{aligned}
$$

In the flight delay problem, the quantifier NOT(most) will also occur. It can be interpreted as the minority of the whole, whereas the quantifier most has the sense of the majority of the whole. The formula

$$
\begin{gathered}
\text { majority }+ \text { minority }=\text { the whole }, \\
\text { most }+\operatorname{NOT}(\text { most })=\text { the whole }, \\
m+\operatorname{NOT}(m)=1
\end{gathered}
$$

expresses the dependence between both the quantifiers.

The RDM model of the quantifier NOT (most)

$$
\begin{aligned}
& \operatorname{NOT}(\text { most }): \\
& \begin{aligned}
\operatorname{NOT}(m)= & 1-m \\
= & \frac{1}{6}(3-2 \mu)\left(1-\alpha_{m}\right), \quad \alpha_{m} \in[0,1] .
\end{aligned}
\end{aligned}
$$

can be obtained from (11) and (13). Membership functions of most and NOT(most) are shown in Fig. 13

It should be noted that the RDM variable $\alpha_{m}$ enables the modeling of coupled values of variables $m$ and $\operatorname{NOT}(m)$ characterizing the quantifiers most and NOT(most). This is not possible in the case of Moore's interval arithmetic.

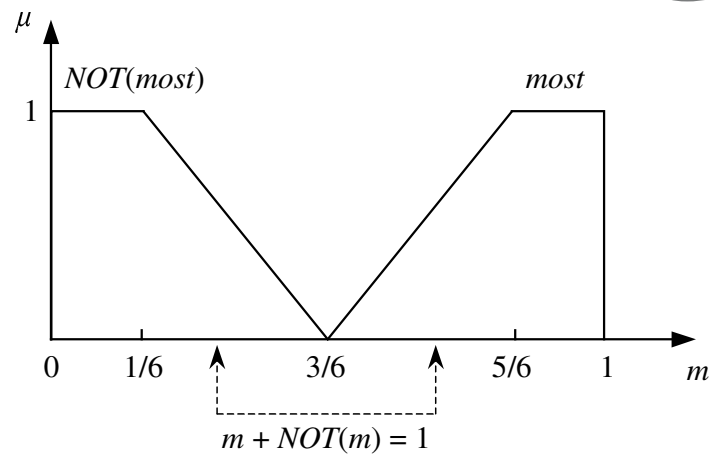

Fig. 13. Membership function of the linguistic quantifier most and of the coupled function NOT(most) being the antonym of most.

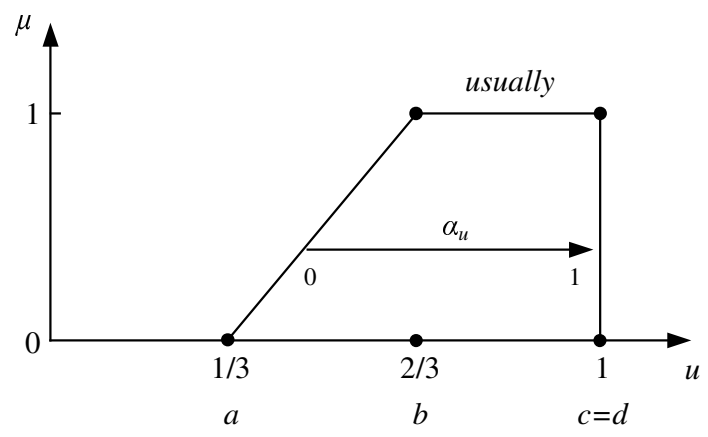

Fig. 14. Membership function of the linguistic quantifier usually.

In the flight delay problem, the linguistic quantifier usually will also be used. The notion of this quantifier is not clear, because it is connected to the notion of 'usuality', (Zadeh, 1996c). According to Zadeh, usuality is a concept that can be expressed not necessarily by one but by a few similar quantifiers such as most, almost always, usually, high probability, etc., i.e., by quantifiers that describe the usual value of variable $X$ : usually $(X$ is $F$ ). In many of his papers and conference opening lectures, Prof. Zadeh has presented a membership function of usually and used the word 'quantifier' for it. Taking this fact into account, we have solved the flight delay problem according to its original formulation given by Zadeh. The membership function $\mu(u)$ of this quantifier represents its understanding by the PIS (Zadeh, 2004) and is given in Fig. 14

The formula

$$
\text { usually : } u=\frac{1}{3}\left[(1+\mu)+(2-\mu) \alpha_{u}\right], \quad \alpha_{u} \in[0,1]
$$

gives the inverse, fuzzy RDM model of the quantifier $u s u$ ally. The variable $u$ can be interpreted as an indication of frequency. Its value, e.g., $u=0.70$, means that in $70 \%$ of observed days the event "most UA aeroplanes departed punctually" happened. The support of usually shown in 


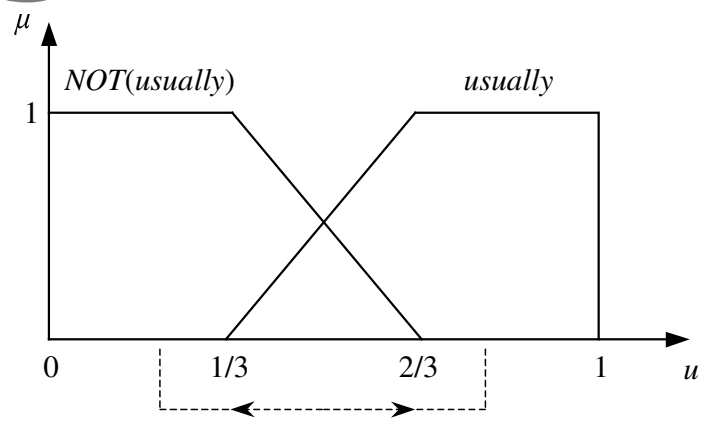

Fig. 15. Membership functions of the quantifiers usually and NOT (usually) being the antonym of usually in accordance with the relation 17 .

Fig. 14 contains all values of $u \in[1 / 3,1]$.

The following formula determines $\mu$-cuts on three levels of membership $(1,0.5$, and 0$)$ :

$$
\begin{aligned}
\mu=1: & u(\mu=1)=\frac{2}{3}+\frac{1}{3} \alpha_{u}, \quad \alpha_{u} \in[0,1], \\
\mu=0.5: & u(\mu=0.5)=\frac{1.5}{3}+\frac{1.5}{3} \alpha_{u}, \\
\mu=0: & u(\mu=0)=\frac{1}{3}+\frac{2}{3} \alpha_{u} .
\end{aligned}
$$

Below, symbolic dependences concerning the quantifiers usually and NOT(usually) are given

$$
\begin{gathered}
\text { usually }+\operatorname{NOT}(\text { usually })=\text { always }, \\
u+\operatorname{NOT}(u)=1 .
\end{gathered}
$$

The formula (17) states that the values of $u$ and $N O T(u)$ are fully correlated (Fig. 15). On the basis of (15) and (17), the inverse membership function of $\operatorname{NOT}(u)$ is obtained:

$$
\operatorname{NOT}(u)=1-u=\frac{1}{3}(2-\mu)\left(1-\alpha_{u}\right), \quad \alpha_{u} \in[0,1] .
$$

For the chosen $\mu$-levels $0,0.5$ and $1, \mu$-cuts of the function (18) given by

$$
\begin{aligned}
\mu=1: & \operatorname{NOT}(u)=\frac{1}{3}\left(1-\alpha_{u}\right), \quad \alpha_{u} \in[0,1], \\
\mu=0.5: & \operatorname{NOT}(u)=\frac{1.5}{3}\left(1-\alpha_{u}\right), \\
\mu=0: & \operatorname{NOT}(u)=\frac{2}{3}\left(1-\alpha_{u}\right) .
\end{aligned}
$$

are achieved. The linguistic values usually, NOT(usually), most, and NOT(most) form a set of four possible fuzzy events that may occur at the airport due to the departures of the United Airlines planes (Fig. 16).

Denote by $P_{\text {punct }}$ and $P_{\text {unp }}$ the probabilities of punctual and unpunctual flights, respectively. As the question in the flight delay problem is "What is the probability that my flight will be delayed?", the value of the probability $P_{u n p}$ is to be determined. This value consists of the probability of events $P_{3}$ and $P_{4}$, shown in Fig. 16. Why? Because on the given day the following four (fuzzy) events may happen:

- Most or a majority of UA aeroplanes will depart today punctually with the probability expressed by the linguistic quantifier usually (Event 1).

- $\operatorname{NOT}(m o s t)$ or a minority of UA aeroplanes will depart today punctually with the probability NOT(usually) (Event 2).

- Most or a majority of UA aeroplanes will depart today unpunctually with the probability NOT(usually) (Event 3).

- NOT $(m o s t)$ or a minority of UA aeroplanes will depart today unpunctually with the probability $u s u$ ally (Event 4).

Unpunctual flights happen in the case of Events 3 and 4 . Thus, the probability $P_{\text {unp }}$ of unpunctual departure is expressed by

$$
\begin{aligned}
P_{\text {unp }} & =P_{3}+P_{4} \\
& =N O T(u) \cdot m+u \cdot \operatorname{NOT}(m) \\
& =(1-u) m+u(1-m) \\
& =m+u-2 m u .
\end{aligned}
$$

On the basis of (11), (14), (15), (18) and (20), we get

$$
\begin{aligned}
P_{\text {unp }}= & \frac{1}{18}\left\{(2-\mu)\left(1-\alpha_{u}\right)\left[(3+2 \mu)+(2-2 \mu) \alpha_{m}\right]\right. \\
& \left.+\left[(1+\mu)+(2-\mu) \alpha_{u}\right](3-2 \mu)\left(1-\alpha_{m}\right)\right\}, \\
& \alpha_{u} \in[0,1], \quad \alpha_{m} \in[0,1], \quad \mu \in[0,1] . \quad
\end{aligned}
$$

This determines the 3D solution set $\Omega_{1}$ being also a 3D granule: $P_{\text {unp }}=f\left(\mu, \alpha_{m}, \alpha_{u}\right)$.

Further on, ranges of the possible probability $\left[P_{\text {unp min }}, P_{\text {unp max }}\right]$ for three levels of membership $\mu$, i.e., $0,0.5$, and 1 , will be determined. Similarly, ranges for any $\mu$-cut can be found.

Level $\mu=1$. For the level $\mu=1$, the formula (21) can be simplified to

$$
P_{\text {unp }}(\mu=1)=\frac{1}{18}\left(7-\alpha_{m}-4 \alpha_{u}-2 \alpha_{m} \alpha_{u}\right) .
$$

A function can have its extremes either at zeros of its derivatives or on the domain boundaries. An examination of the derivatives of (22) gives

$$
\begin{aligned}
& \frac{\mathrm{d} P_{\text {unp }}}{\mathrm{d} \alpha_{m}}=\frac{1}{18}\left(-1-2 \alpha_{u}\right)=0, \quad \alpha_{u \text { opt }}=-0.5 \notin[0,1], \\
& \frac{\mathrm{d} P_{\text {unp }}}{\mathrm{d} \alpha_{u}}=\frac{1}{18}\left(-4-2 \alpha_{m}\right)=0, \quad \alpha_{m \text { opt }}=-2 \notin[0,1] .
\end{aligned}
$$



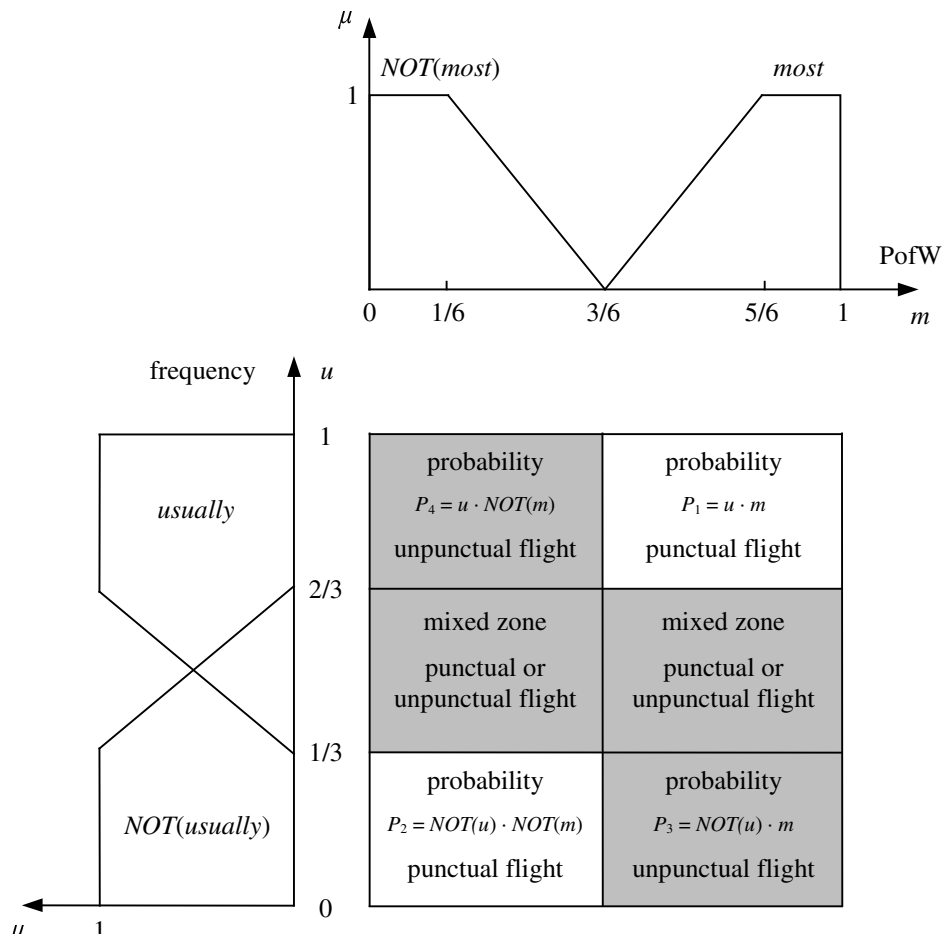

Fig. 16. Set of four possible fuzzy events which may occur at the airport due to departures of planes today.

Table 2. Results of the examination of the boundaries for the function [22, $P_{\text {unp }}(\mu=1) \in[0,7 / 18]$.

\begin{tabular}{|c||cccc|}
\hline$\alpha_{m}$ & 0 & 0 & 1 & 1 \\
$\alpha_{u}$ & 0 & 1 & 0 & 1 \\
\hline$P_{\text {unp }}$ & $\frac{7}{18}$ & $\frac{3}{18}$ & $\frac{6}{18}$ & 0 \\
\hline
\end{tabular}

The results 23 mean that extremes of the function (22) do not lie inside the domain $\alpha_{u} \times \alpha_{m}=[0,1] \times$ $[0,1]$. Therefore, the boundaries of the domain are to be examined. Table 2 shows results of this examination for boundary values of RDM variables $\alpha_{u}$ and $\alpha_{m}$.

Level $\boldsymbol{\mu}=\mathbf{0 . 5}$. For this level, (21) simplifies to

$$
P_{\text {unp }}(\mu=0.5)=\frac{3}{18}\left(3-\alpha_{u}-2 \alpha_{m} \alpha_{u}\right) .
$$

The examination of the derivatives of $(24)$ gives

$$
\frac{\mathrm{d} P_{\text {unp }}}{\mathrm{d} \alpha_{m}}=\frac{3}{18}\left(-2 \alpha_{u}\right)=0, \quad \alpha_{u \text { opt }}=0,
$$

and

$$
\begin{aligned}
\frac{\mathrm{d} P_{\text {unp }}}{\mathrm{d} \alpha_{u}} & =\frac{3}{18}\left(-1-2 \alpha_{m}\right)=0, \\
\alpha_{m \text { opt }} & =-0.5 \notin[0,1] .
\end{aligned}
$$

The point determined by the vector $\left(\alpha_{m \text { opt }}, \alpha_{u \text { opt }}\right)=(0,-0.5)$ does not lie in the solution
Table 3. Results of the examination of the boundaries for the function (24) $P_{\text {unp }}(\mu=0.5) \in[0,9 / 18]$.

\begin{tabular}{|c||cccc|}
\hline$\alpha_{m}$ & 0 & 0 & 1 & 1 \\
$\alpha_{u}$ & 0 & 1 & 0 & 1 \\
\hline$P_{\text {unp }}$ & $\frac{9}{18}$ & $\frac{9}{18}$ & $\frac{6}{18}$ & 0 \\
\hline
\end{tabular}

domain $[0,1] \times[0,1]$. Thus, the extremes should be sought at the boundaries of the domain $\alpha_{u} \times \alpha_{m}$, Table 3 .

Figure 17 shows the $\Omega_{1}$ set - the 3D solution granule determining the probability of a delayed flight $P_{\text {unp }}$ on the level $\mu=0.5$.

The $\Omega_{1}$ granule of the precise solution of Eqn. (24) shown in Fig. 17, is defined by

$$
\begin{gathered}
\Omega_{1}=\left\{P_{\text {unp }}\left(\mu=0.5, \alpha_{m}, \alpha_{u}\right) \mid\right. \\
P_{\text {unp }}=\frac{3}{18}\left(3-\alpha_{u}-2 \alpha_{m} \alpha_{u}\right), \\
\left.\alpha_{m}, \alpha_{u} \in[0,1]\right\} .
\end{gathered}
$$

The interval (Moore's arithmetic) solution $\Omega_{2}$ : $P_{\text {unp }}(\mu=0.5) \in[0,9 / 18]$ is not the precise solution but only a one-dimensional representation of the precise $\Omega_{1}$ 


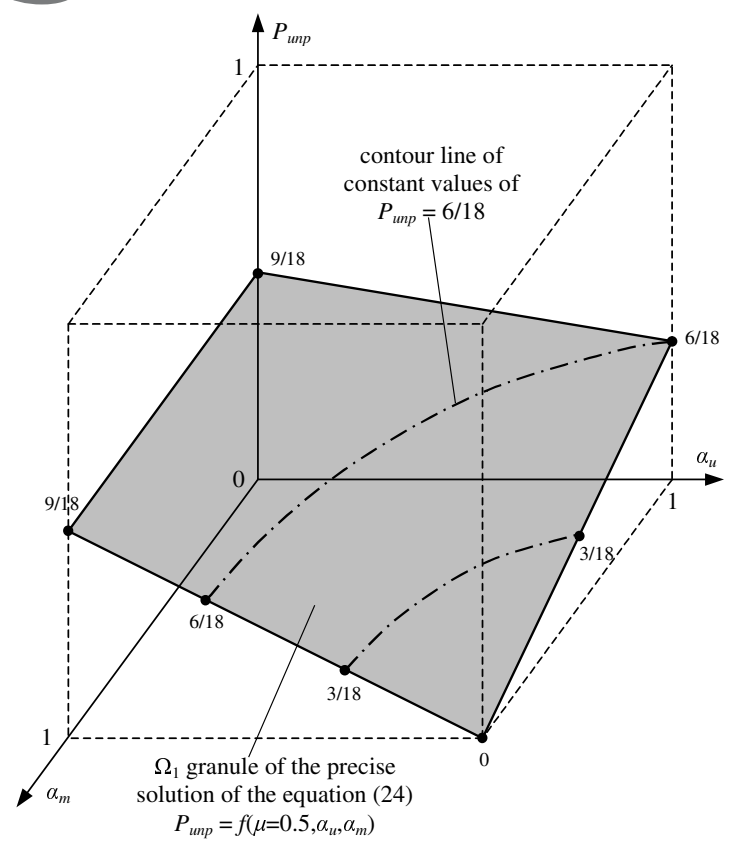

Fig. 17. Solution visualization of Eqn. 24 determining the probability of a delayed flight $P_{\text {unp }}$ for the membership level $\mu=0.5$.

solution (24) for the level $\mu=0.5$. We have

$$
\begin{aligned}
\Omega_{2}=\{ & P_{\text {unp }}\left(\mu=0.5, \alpha_{m}, \alpha_{u}\right) \mid \\
& P_{\text {unp }} \in\left[\min \left(P_{\text {unp }}\left(\mu=0.5, \alpha_{m}, \alpha_{u}\right)\right),\right. \\
& \left.\max \left(P_{\text {unp }}\left(\mu=0.5, \alpha_{m}, \alpha_{u}\right)\right)\right], \\
& \left.\alpha_{m}, \alpha_{u} \in[0,1]\right\} .
\end{aligned}
$$

The $\Omega_{2}$ solution for the level $\mu=0.5$ is displayed in Fig. 18.

The comparison of Figs. 17 and 18 allows the understanding of the difference between the $\Omega_{1}$ and $\Omega_{2}$ solutions. The $\Omega_{1}$ solution based on the formulas (21) and (24) containing RDM variables $\alpha_{m}$ and $\alpha_{u}$ is the precise solution. The $\Omega_{2}$ solution called in the literature (Lyashko, 2005; Wang and Qiu, 2013) the "interval hull of the united solution set $\Omega_{1}$ " states the spread of the precise solution. However, for practical purposes it may often be sufficient.

Level $\boldsymbol{\mu}=\mathbf{0}$. For the level $\mu=0$, the formula 21) determining probability $P_{\text {unp }}$ of a delayed flight simplifies to

$$
\begin{aligned}
& P_{\text {unp }}(\mu=0)=\frac{1}{18}\left(9+3 \alpha_{m}-12 \alpha_{m} \alpha_{u}\right) \\
& \alpha_{m}, \alpha_{u} \in[0,1] .
\end{aligned}
$$

To find the extremes of the probability, both

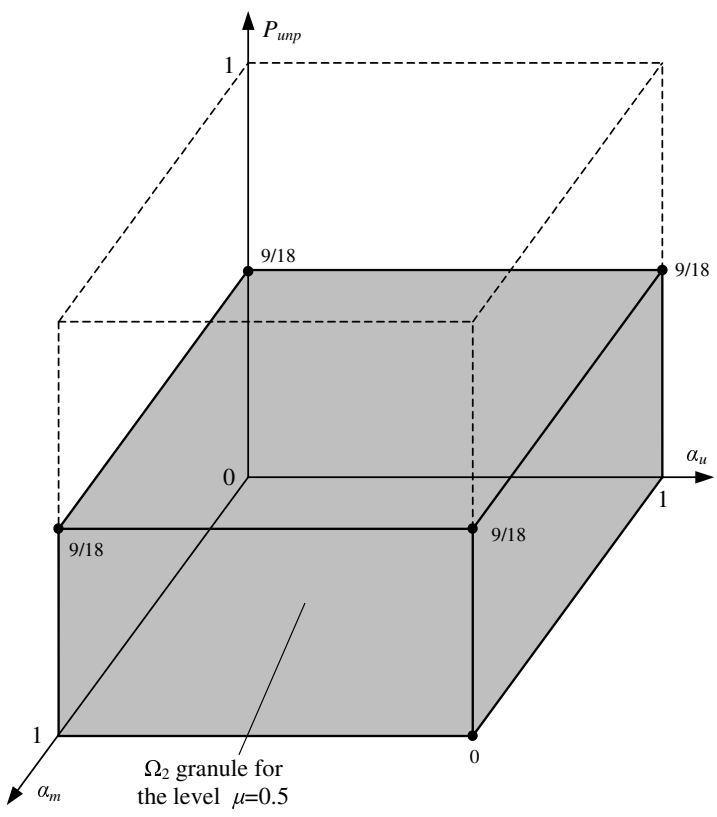

Fig. 18. Visualization of Moore's $\Omega_{2}$ solution of the flight delay problem for the membership level $\mu=0.5$.

Table 4. Results of the examination of the probability $P_{\text {unp }}$ of the flight delay for the membership level $\mu=0$.

\begin{tabular}{|c||cccc|}
\hline$\alpha_{m}$ & 0 & 0 & 1 & 1 \\
$\alpha_{u}$ & 0 & 1 & 0 & 1 \\
\hline$P_{\text {unp }}$ & $\frac{9}{18}$ & $\frac{9}{18}$ & $\frac{12}{18}$ & 0 \\
\hline
\end{tabular}

derivatives of the probability

$$
\begin{aligned}
& \frac{\mathrm{d} P_{\text {unp }}}{\mathrm{d} \alpha_{m}}=\frac{1}{18}\left(3-12 \alpha_{u}\right)=0, \quad \alpha_{u \text { opt }}=0.25, \\
& \frac{\mathrm{d} P_{\text {unp }}}{\mathrm{d} \alpha_{u}}=\frac{1}{18}\left(-12 \alpha_{m}\right)=0, \quad \alpha_{m \text { opt }}=0
\end{aligned}
$$

and the probability values on the boundaries of its domain (Table 4) were examined.

The examination showed that for all the three membership levels $(0,0.5$ and 1$)$ the probability extremes lie on the boundaries of the domain $\alpha_{m} \times \alpha_{u}, \alpha_{u} \in$ $[0,1], \alpha_{m} \in[0,1]$. Figure 19 shows all three precise 3D-solutions $\Omega_{1}(\mu=0), \Omega_{1}(\mu=0.5)$ and $\Omega_{1}(\mu=1)$ for particular $\mu$-cuts.

The membership function of the $\Omega_{2}$ solution of the 'interval hull' type, 27), is presented in Fig. 20

The membership function $\mu\left(P_{\text {unp }}\right)$ in Fig. 20 can be interpreted as the flight delay probability about 0.27 or also as approximately below 0.5. The minimal and maximal values of the probability $P_{\text {unp }}$ for the membership function $\mu\left(P_{\text {unp }}\right)$ shown in Fig. 20 can be 


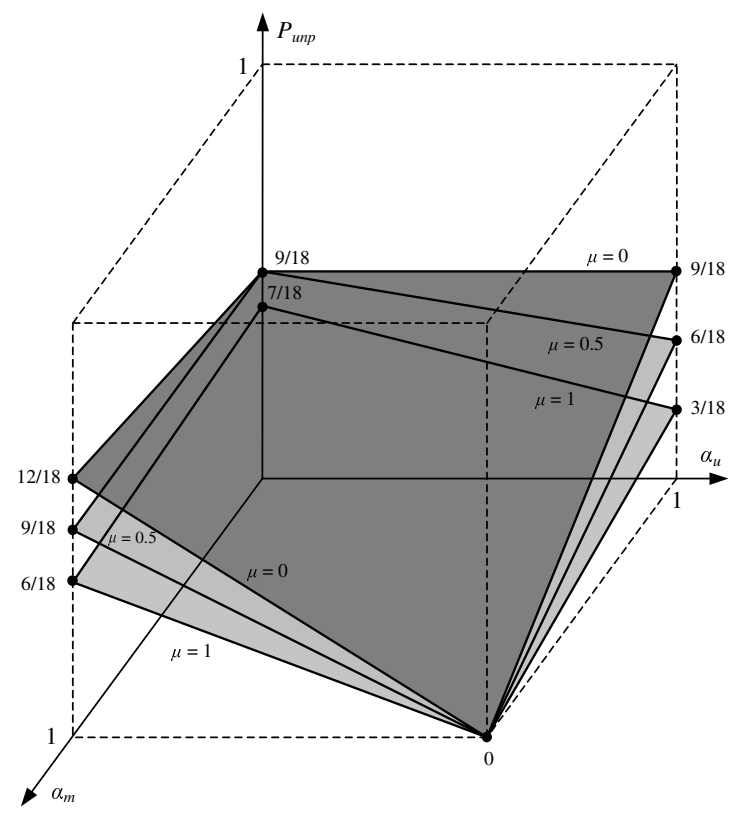

Fig. 19. 3D granules of 3 precise RDM solutions $\Omega_{1}(\mu=0)$, $\Omega_{1}(\mu=0.5), \Omega_{1}(\mu=1)$ for particular $\mu$-cuts.

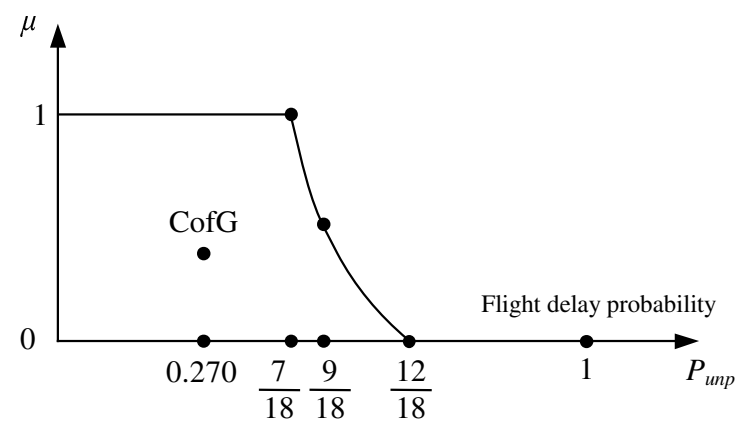

Fig. 20. Membership function of the flight delay probability $P_{\text {unp }}$ achieved with the use of RDM inverse models of membership functions. CofG is the center of gravity.

determined using

$$
\begin{aligned}
& \min \left(P_{\text {unp }}(\mu)\right)=\min \{ \frac{1}{18}\left(9+2 \mu-4 \mu^{2}\right), \frac{6}{18}(2-\mu), \\
&\left.\frac{3}{18}(3-2 \mu), 0\right\} \\
& \max \left(P_{\text {unp }}(\mu)\right)=\max \{ \frac{1}{18}\left(9+2 \mu-4 \mu^{2}\right), \frac{6}{18}(2-\mu), \\
&\left.\frac{3}{18}(3-2 \mu), 0\right\},
\end{aligned}
$$

The formulas (30) were achieved on the basis of the formula (21) for various combinations of boundary values of RDM variables $\alpha_{u}$ and $\alpha_{m}$ (Table 5).

On the basis of experience gained during solving Zadeh's flight delay problem and other similar issues, we can give the following, general method of $\mathrm{CWW}$ problems solving:
Table 5. Formulas of the delay probability $P_{\text {unp }}(\mu)$ for 4 boundary combinations of RDM variables $\alpha_{u} \in[0,1]$ and $\alpha_{m} \in[0,1]$.

\begin{tabular}{|c||cccc|}
\hline$\alpha_{m}$ & 0 & 0 & 1 & 1 \\
\hline$\alpha_{u}$ & 0 & 1 & 0 & 1 \\
\hline$P_{\text {unp }}(\mu)$ & $\frac{1}{18}\left(9+2 \mu-4 \mu^{2}\right)$ & $\frac{6}{18}(2-\mu)$ & $\frac{3}{18}(3-2 \mu)$ & 0 \\
\hline
\end{tabular}

1. Determine variables and linguistic values of variables occurring in the problem.

2. Determine general formulas enabling problem solving.

3. Determine RDM models of inverse membership functions of linguistic quantifiers occurring in the problem.

4. Use inverse RDM models of linguistic quantifiers and formulas for problem solving, for determining the precise, multidimensional problem solution $\Omega_{1}$.

5. On the basis of the precise multidimensional solution $\Omega_{1}$, determine the interval hull solution $\Omega_{2}$.

\section{Testing the achieved solution}

Computing with words imitates the human way of reasoning, thinking and problem solving with linguistic information. Thus, the natural way of testing achieved results in the flight delay problem is their comparison with mental solution results of this issue provided by people. At the Faculty of Computer Science of our home university, a study with the participation of 54 students was conducted. Each student received the following description of the problem:

Let us assume that you are waiting for your airplane and an airport officer informs you that "Usually most United Airlines flights leave on time." How would you evaluate the probability that your today flight will be delayed?

Variant 1. Use linguistic values: probability 'below 0.5 ' or 'above 0.5'.

Variant 2. Use one of linguistic values: 'low', 'low or medium', 'medium', 'medium or high', 'high' probability.

Figure 21 shows the histogram of student's answers for Variant 1.

As can be seen in Fig. 21, the center of gravity as the singleton representation of the histogram has the position $P_{\text {unp }}=0.306$, whereas the result of computing with words is $P_{\text {unp }}=0.270$. Thus, people evaluated the probability 


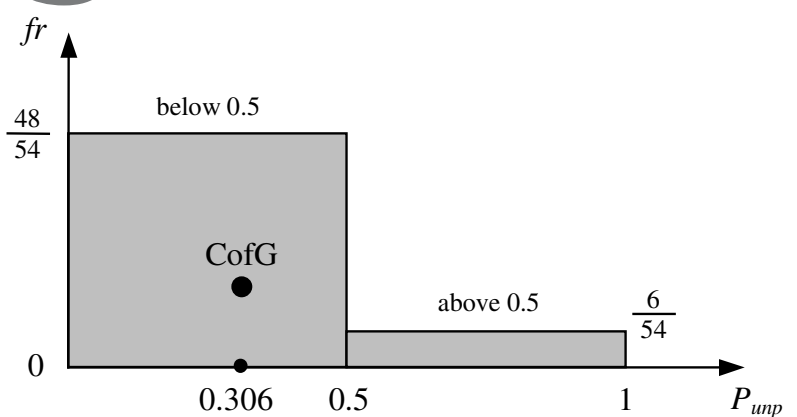

Fig. 21. Histogram of probability evaluations 'below 0.5 ' and 'above 0.5 ' of a delayed flight as a result of the examination of 54 persons. $f r$ is the response frequency, CofG is the center of gravity.

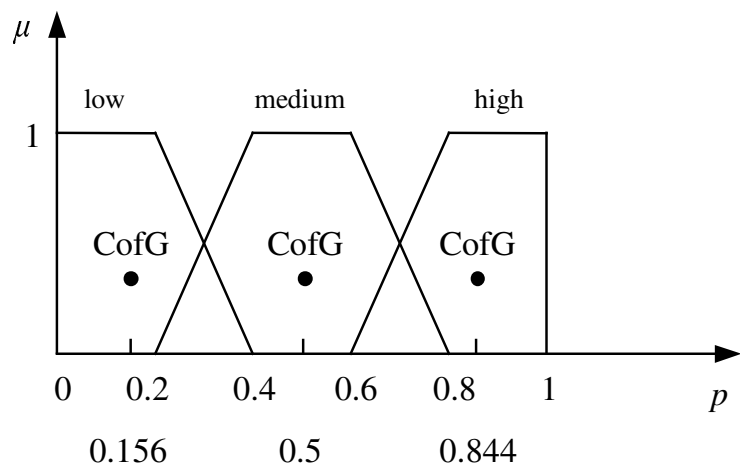

Fig. 22. Membership functions of the linguistic values low, medium and high probability of a delayed flight with the positions of their centers of gravity.

of a delayed flight similarly but a little higher than $\mathrm{CwW}$, though the difference is small. In Variant 2, students could chose one of 5 linguistic values: the probability 'low', 'low or medium', 'medium', 'high or medium', 'high'. 22 out of 54 persons evaluated the probability as 'low', 19 as 'low or medium', 12 as 'medium', and 1 person as high. Figure 22 shows the membership function of the values low, medium, high probability.

On the basis of answers of 54 persons, a position of the gravity center equal to 0.335 was obtained. This value, having the meaning of the expected delay probability, is also greater than the result 0.270 achieved with the $\mathrm{CwW}$ method. Thus, the question arises: "What is the reason for this difference?", "Why do people evaluate the delay probability higher than the computing with words?" and "Is $\mathrm{CwW}$ more precise than people or are people more precise in their mental calculations?".

To explain it, we should recall the information provided for students and the stated question: "Usually most flights of UA from San Francisco leave on time. What is the probability that my today flight will be delayed?". The human mind analyzes only the information directly given in the statement "Usually most flights of UA leave on time" and on this basis intuitively evaluates, first the probability $P_{\text {punct }}$ of the punctual flight that is given symbolically by

$$
P_{\text {punct }}=\text { usually } \cdot \text { most },
$$

and next the probability of delayed flight $P_{\text {unp }}$ given by (32)

$$
P_{\text {unp }}=1-P_{\text {punct }}=1-\text { usually } \cdot \text { most. }
$$

Both (31) and (32) are partially incorrect. None of the persons asked detected that if airplanes leave punctually in the situation usually - most, then they also leave punctually in the situation NOT(usually). NOT (most) (Fig. 16). Most people are even unable to understand this situation because it is described by double negation (people tend to focus on the ratio of days on which most airplanes leave on time and on the ratio of airplanes which leave on time on a single day). Because the correct probability value of the punctual flight is determined by the formula

$$
\begin{aligned}
P_{\text {punct }}= & \text { usually } \cdot \text { most } \\
& +\operatorname{NOT}(\text { usually }) \cdot \operatorname{NOT}(\text { most }),
\end{aligned}
$$

the correct probability value of the unpunctual flight is determined by

$$
\begin{aligned}
P_{\text {unp }}= & 1-P_{\text {punct }} \\
= & 1-\text { usually } \cdot \text { most } \\
& -\operatorname{NOT}(\text { usually }) \cdot \operatorname{NOT}(\text { most }) .
\end{aligned}
$$

The comparison of (34) with (32) clearly shows why people evaluate the flight delay probability a little higher ( 0.306 or 0.335 ) than the $\mathrm{CwW}$ method, which evaluates it as 0.270 . The segment NOT(usually) $\cdot \operatorname{NOT}$ (most) is not subtracted from 1 in 32). The presented flight delay problem makes us aware that people use in their mental calculations only direct perceptions, rather than hidden perceptions, which are important but cannot be perceived directly in an easy way and have to be presumed or suspected. However, each perception means one event possibility - the possibility of informing when the event can occur. Probably people intuitively feel and realize the insufficiency of their analytical possibilities and compensate this insufficiency by an increased filling of the risk and by risk aversion detected and investigated by Nobel Prize winners Kahneman and Tversky (2000). The above means that the practical usability of computing with words can be considerable and that $\mathrm{CwW}$ can increase reasoning precision.

\section{Conclusions}

The paper presented new inverse membership functions based on RDM interval arithmetic. These inverse 
functions model not only boundaries but also the inner space of membership functions and allow effective computing with words. Their effectiveness was shown using the flight delay problem formulated by Zadeh. The obtained solution is credible and was compared with mental solutions provided by a 54-student group. Both results are similar, though the research demonstrates that people overestimate the probability of flight delay. The paper also presents the reasons behind such a situation. Generally, results presented in the paper allow optimistic evaluation of the abilities of computing with words as a practical science area.

\section{References}

Aliev, R., Pedrycz, W., Fazlollahi, B., Huseynov, O., Alizadeh, A. and Guirimov, B. (2012). Fuzzy logic-based generalized decision theory with imperfect information, Information Sciences 189: 18-42.

Batyrshin, I. (2002). On granular derivatives and the solution of a granular initial value problem, International Journal of Applied Mathematics and Computer Science 12(3): 403-410.

Batyrshin, I. and Wageknecht, M. (2002). Towards a linguistic description of dependences in data, International Journal of Applied Mathematics and Computer Science 12(3): 391-401.

Cao, T. (2003). Conceptual graphs for modelling and computing with generally quantified statements, in J. Lawry, J. Shanahan and A. Ralescu (Eds.), Modelling with Words, Springer, Berlin/Heidelberg, pp. 80-101.

De Cock, M. and Kerre, E.E. (2002). A context-based approach to linguistic hedges, International Journal of Applied Mathematics and Computer Science 12(3): 371-382.

Dymova, L. (2011). Soft Computing in Economics and Finance, Springer Verlag, Berlin/Heidelberg.

Gemeinder, M. (2002). Imposing restrictions on density functions utilised in computing with words, International Journal of Applied Mathematics and Computer Science 12(3): 383-390.

Grzegorzewski, P. and Hryniewicz, O. (2002). Computing with words and life data, International Journal of Applied Mathematics and Computer Science 12(3): 337-345.

Hansen, E. (1975). A generalized interval arithmetic, in K. Nickel (Ed.), Interval Mathematics, Lecture Notes in Computer Science, Vol. 29, Springer Verlag, Berlin/Heidelberg, pp. 7-18.

Hanss, M. (2005). Applied Fuzzy Arithmetic, Springer Verlag, Berlin/Heidelberg.

Herrera, F., López, E., Mendaña, C. and Rodríguez, M. (1999). A linguistic decision model to suppliers selection in international purchasing, Computing with Words in Information/Intelligent Systems 2, Physica-Verlag, Heidelberg, pp. 500-524.
Kacprzyk, J. and Zadrożny, S. (1999). The paradigm of computing with words in intelligent database querying, Computing with Words in Information/Intelligent Systems 2, Physica-Verlag, Heidelberg, pp. 383-398.

Kacprzyk, J. and Zadrożny, S. (2002). Linguistic data summaries: Towards an increased role of natural language in data mining, Proceedings of the 8th IEEE International Conference on Methods and Models in Automation and Robotics, Szczecin, Poland, pp. 121-126.

Kacprzyk, J. and Zadrożny, S. (2010). Computing with words is an implementable paradigm: Fuzzy queries, linguistic data summaries and natural language generation, IEEE Transactions on Fuzzy Systems 18(3): 461-472.

Kahnemann, D. and Tversky, A. (2000). Choices, Values and Frames, Cambridge University Press, Russel Sage Foundation, Cambridge.

Kaufmann, A. and Gupta, M. (1991). Introduction to Fuzzy Arithmetic, Van Nostrand Reinhold, New York, NY.

Landowski, M. (2014). Differences between Moore's and RDM interval arithmetic, Proceedings of the 13th International Workshop on Intuitionistic Fuzzy Sets and Generalized Nets, Warsaw, Poland, pp. 331-340.

Lawry, J. (2006). Modelling and Reasoning with Vague Concepts, Studies in Computational Intelligence, Vol. 12, Springer-Verlag, New York, NY.

Lyashko, M. (2005). The optimal solution of an interval system of linear algebraic equations, Reliable Computing 11(2): 105-127.

Mendel, J.M. (2002). An architecture for making judgments using computing with words, International Journal of Applied Mathematics and Computer Science 12(3): 325-335.

Moore, R. (1966). Interval Analysis, Prentice Hall, Englewood Cliffs, NJ.

Moore, R., Kearfott, R. and Cloud, M. (2009). Introduction to Interval Analysis, Society for Industrial and Applied Mathematics, Philadelphia, PA.

Pedrycz, W. and Gomide, F. (2007). Fuzzy Systems Engineering: Toward Human-Centric Computing, Wiley Interscience, Hoboken, NJ.

Piegat, A. (2001). Fuzzy Modeling and Control, Physica Verlag, Heidelberg/New York, NY.

Piegat, A. and Landowski, M. (2012). Is the conventional interval-arithmetic correct?, Journal of Theoretical and Applied Computer Science 6(2): 27-44.

Piegat, A. and Landowski, M. (2013a). Multidimensional approach to interval-uncertainty calculations, in $\mathrm{K}$. Atanassov et al. (Eds.), New Trends in Fuzzy Sets, Intuitionistic Fuzzy Sets, Generalized Nets and Related Topics, System Research Institute of the Polish Academy of Sciences, Warsaw, pp. 137-152.

Piegat, A. and Landowski, M. (2013b). Two interpretations of multidimensional RDM interval arithmetic: Multiplication and division, International Journal of Fuzzy Systems 15(4): 486-496. 
Piegat, A. and Tomaszewska, K. (2013). Decision-making under uncertainty using info-gap theory and a new multidimensional RDM interval-arithmetic, Electrical Review 88(8): 71-76.

Rajati, M. and Mendel, J. (2012). Lower and upper probability calculations using compatibility measures for solving Zadeh's challenge problems, Proceedings of the IEEE International Conference on Fuzzy Systems, Brisbane, Australia, pp. 1-8.

Rajati, M., Mendel, J. and Wu, D. (2011). Solving Zadeh's Magnus challenge problem on linguistic probabilities via linguistic weighted averages, Proceedings of the IEEE International Conference on Fuzzy Systems, Taipei, Taiwan, pp. 2177-2184.

Sevastjanov, P. and Dymova, L. (2009). A new method for solving interval and fuzzy equations: Linear case, Information Sciences 17: 925-937.

Tomaszewska, K. (2014). The application of horizontal membership function to fuzzy arithmetic operations, Proceedings of the 11th Polish Conference of Students and Young Scientists, Szczecin, Poland, pp. 1-8.

Tomaszewska, K. and Piegat, A. (2014). Application of the horizontal membership function to the uncertain displacement calculation of a composite massless rod under a tensile load, Proceedings of the International Conference on Advanced Computer Systems, Międzyzdroje, Poland, pp. 63-72.

Türkşen, I. (2007). Meta-linguistic axioms as a foundation for computing with words, Information Sciences 177(2): 332-359.

Wang, C. and Qiu, Z. (2013). Equivalent method of accurate solution to linear interval equations, Applied Mathematics and Mechanics 34(8): 1031-1042.

Zadeh, L. (1996a). Fuzzy logic = computing with words, IEEE Transactions on Fuzzy Systems 4(2): 103-111.

Zadeh, L. (1996b). Linguistic characterization of preference relations as a basis for choice in social systems, in G. Klir and B. Yuan (Eds.), Fuzzy Sets, Fuzzy Logic, and Fuzzy Systems, World Scientific Publishing Co., River Edge, NJ, pp. 336-354.

Zadeh, L. (1996c). Outline of a theory of usuality based on fuzzy logic, in G. Klir and B. Yuan (Eds.), Fuzzy Sets, Fuzzy Logic, and Fuzzy Systems: Selected Papers by Lotfi A. Zadeh, World Scientific Publishing Co., River Edge, NJ, pp. 694-712.

Zadeh, L. (2001). A new direction in AI-toward a computational theory of perceptions, AI Magazine 22(1): 73-84.

Zadeh, L.A. (2002). From computing with numbers to computing with words-from manipulation of measurements to manipulation of perceptions, International Journal of Applied Mathematics and Computer Science 12(3): 307-324.

Zadeh, L. (2004). A note on web intelligence, world knowledge and fuzzy logic, Data \& Knowledge Engineering 50(3): 291-304.
Zadeh, L. (2005). Toward a generalized theory of uncertainty (GTU), Special lecture, Polish Academy of Sciences, Warsaw.

Zadeh, L. (2006a). Generalized theory of uncertainty (GTU)—principal concepts and ideas, Computational statistics \& Data Analysis 51(1): 15-46.

Zadeh, L. (2006b). A new frontier in computation-computation with information described in natural language, International Conference ICAISC, Zakopane, Poland, (plenary lecture).

Zadeh, L. (2009). Computing with words and perceptions-a paradigm shift, 2009 IEEE International Conference on Information Reuse and Integration, Las Vegas, NV, USA, pp. VIII-X.

Zadeh, L. and Kacprzyk, J. (Eds.) (1999). Computing with Words in Information/Intelligent Systems 1: Foundations, Studies in Fuzziness and Soft Computing, Vol. 33, Physica-Verlag, Heidelberg.

Zhou, C. (2002). Fuzzy-arithmetic-based Lyapunov synthesis in the design of stable fuzzy controllers: A computing-with-words approach, International Journal of Applied Mathematics and Computer Science 12(3): 411-421.

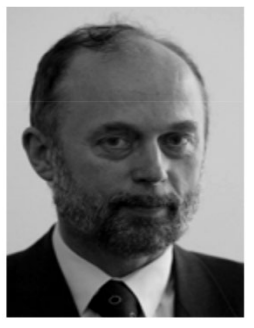

theory.
Andrzej Piegat received a Ph.D.degree in modeling and control of production systems from the Technical University of Szczecin, Poland, in 1979, a D.Sc. degree in control of underwater vehicles from the University of Rostock, Germany, in 1998, and a professorial title in 2001. Presently he is a professor at the West Pomeranian University of Technology, Poland. His current research is focused on uncertainty theory, fuzzy logic, computing with words and info-gap

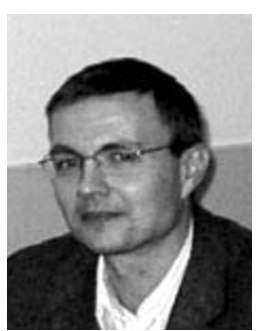

Marcin Pluciński received an M.Sc. degree from the Faculty of Marine Technology of the Szczecin University of Technology in 1988 and a Ph.D. degree from the Faculty of Computer Systems and Information Technology of the same university in 1996. Since 1996 he has been working at the Faculty of Computer Systems and Information Technology of the West Pomeranian University of Technology. His research interests cover mobile robotics, reinforcement learning, fuzzy modeling and control, and computing with words.

Received: 3 February 2014

Revised: 24 September 2014

Re-revised: 17 February 2015 